\title{
A CASE REPORT ON ALLOPURINOL INDUCED DRESS SYNDROME
}

\author{
KARTHIKA S. ${ }^{1}$, KAVYA SURENDRAN ${ }^{1}$, KRISHNAPRIYA M. K. ${ }^{1}$, DONA MARIA JETTO² \\ 1,2Department of Pharmacy Practice, Nirmala College of Pharmacy, Muvattupuzha, Kerala, India 686661 \\ Email: karthikas0016@gmail.com
}

Received: 14 Jun 2021, Revised and Accepted: 28 Jul 2021

\begin{abstract}
Allopurinol-induced DRESS Syndrome or Drug Rash with Eosinophilia and Systemic Symptom is a rare but potentially fatal drug reaction. Fever, rashes, swelling, and hematologic abnormalities, particularly short-term or long-term injury to one or more organs, have been reported by over 0.4 percent of patients following the course of medicine. Here, a 54-year-old female patient was admitted to the hospital with complaints of fever, purpuric rashes over the body with itching mainly in the oral cavity and lips, and the extent to the upper back and lower limbs for three days. She had known complaints of Type 2 Diabetes, Systemic hypertension, Coronary artery disease with recent NSTEMI, Dyslipidaemia, Psychosis (20y), and Chronic kidney disease. She had been taking Allopurinol for the past three months for hyperuricemia. Allopurinol treatment stopped while topical Steroid treatment started along with supportive therapy. After ten days, the rashes improved significantly, allowing her to leave the hospital.
\end{abstract}

Keywords: Allopurinol, Drug reaction with eosinophilia, Systemic symptom, Skin rash

(C) 2021 The Authors. Published by Innovare Academic Sciences Pvt Ltd. This is an open-access article under the CC BY license (https://creativecommons.org/licenses/by/4.0/]

DOI: https://dx.doi.org/10.22159/ijpps.2021v13i9.42430. Journal homepage: https://innovareacademics.in/journals/index.php/ijpps.

\section{INTRODUCTION}

An enzyme inhibitor, Allopurinol, inhibits Xanthine Oxidase and has been effective in reducing urate levels in hyperuricemia patients and relieving Gout symptoms permanently [1]. In 1966, the FDA approved this drug primarily to treat Gout, Tumour Lysis Syndrome. Furthermore, they can prevent calcium nephrolithiasis in hyperuricosuria individuals. The primary component that separates the purine bases and catalyzes the conversion of hypoxanthine to xanthine and xanthine to uric acid is a xanthine oxidase. Uric acid is passed through the body regularly. This chemical insufficiency might be due to a genetic component, with increased purine nourishment ingestion and less catalyst production at some point. Allopurinol increased the action of any drug processed by xanthine oxidase [2]. Acting as an analog of Hypoxanthine, it was mainly used for Gout management by decreasing uric acid production [3].

Allopurinol is a well-tolerated drug with few adverse drug reactions. The mild cutaneous reaction had appeared in almost $2 \%$ of patients [3]. But Allopurinol-induced Hypersensitivity syndrome (AHS) and Drug Rash with Eosinophilia and Systemic Symptom (DRESS) are the rarest but most severe hypersensitivity reactions. The researchers estimated that one in every 260 patients suffers from DRESS Syndrome as an adverse drug reaction of Allopurinol. Within eight weeks of commencing therapy, patients had cutaneous responses, fever, eosinophilia, swelling, renal and hepatic dysfunction, and hepatic damage [3].

The precise mechanism responsible for DRESS Syndrome is unknown, but it may represent the genetic association of HLA$B^{*} 58: 01$ [4]. Although, the most common differential Diagnosis of DRESS Syndrome includes Toxic Epidermal Necrosis, StevenJohnson Syndrome, Eosinophilia, Still's Disease, and Kawasaki Disease [3]. Skin Biopsy is the gold standard for DRESS Syndrome. Discontinuing the substance responsible for its etiology is the first step in implementing a supportive treatment strategy based on the symptoms [5]. Along with that, steroid therapies are also important. The effect of Steroids on the inhibition of Interleukin-5 and Eosinophil accumulation and its dramatic improvement in clinical symptoms make this therapy more beneficial for the patient [3]. Here, we report a case of a 54-year-old female who developed DRESS syndrome as a rare side effect of allopurinol treatment.

\section{CASE REPORT}

A 54-year-old female patient came to the hospital with chief complaints of purpuric rashes over the body. Presented with the itching for the past three days without raised patches, presence of one episode of fever spikes, mild abdominal pain with no history of loose stool, vomiting, travel outside, cough and dyspnea. But her legs appeared swollen mildly. The patient had no loss of consciousness and seizures on admission. The patient had known complaints of Type 2 Diabetes mellitus, Systemic hypertension, Coronary artery disease with recent NSTEMI, Dyslipidaemia, Psychosis (20 y), chronic kidney disease. In addition, the patient was recently diagnosed with hyperuricemia and had been taking Allopurinol 100 mg for the previous three months. The patient was afebrile but had several painful lymph nodes after arrival. On general examination, the patient was conscious, alert, pallor, no icterus, lymphadenopathy, rashes over upper chest, lower limbs, the trunk, sides of the ear, arms, purpuric macules on the legs, tremor, crusting on lips, and generalized tremors. Blood pressure was 160/80 mmHg. Haemoglobin 7.7 g/dl, WBC 2480/cumm, Eosinophil 10\%, Platelet count 100000/cumm, PCV 23.7\%, Serum Urea levels 122.2 mg\%, 97 $\mathrm{mg} \%$, and $106 \mathrm{mg} \%$ respectively, Serum Creatinine $4.15 \mathrm{mg} \%, 3.89$ $\mathrm{mg} \%$, and $4.9 \mathrm{mg} \%$, and Serum Albumin $3.3 \mathrm{gm} \%$ appeared in the blood analysis [table 1].

Table 1: Lab parameters

\begin{tabular}{ll}
\hline Lab parameters & Values \\
\hline Blood pressure & $160 / 80 \mathrm{mmHg}$ \\
Hemoglobin & $7.7 \mathrm{~g} / \mathrm{dl}$ \\
Total WBC & $2480 / \mathrm{cumm}$ \\
Eosinophil & $10 \%$ \\
Platelet Count & $100000 / \mathrm{cumm}$ \\
PCV & $23.7 \%$ \\
Serum Urea Level & $122.2 \mathrm{mg} \%, 96 \mathrm{mg} \%$, and $106 \mathrm{mg} \%$ \\
Serum Creatinine & $4.15 \mathrm{mg} \%, 3.9 \mathrm{mg} \%$ and $4.9 \mathrm{mg} \%$ \\
Serum Albumin & $3.3 \mathrm{~g} \%$ \\
\hline
\end{tabular}

The peripheral smear test showed normocytic normochromic anemia with microcytic hypochromic RBCs, leukopenia, and eosinophilia. It concluded that there was no evidence of dengue fever through a dengue test. No significant regional wall motion abnormalities on ECHO, good LV/RV systolic function, mild mitral valve regurgitation. She was diagnosed with low blood cell counts. The nephrologist recommended stopping Allopurinol, Cardivas, and Magnesium Valproate after the second day and starting Topical 
Steroid therapy alongside supportive therapy. Desowen (Desonide) for local application and Kenocort $0.1 \%$ (Triamcinolone) oromucosal paste was used on the first day and continued for ten days for discharge medication.

\section{DISCUSSION}

Allopurinol is an analog of Hypoxanthine, commonly used in clinical practice for treating hyperuricemia [3]. Patients receiving Allopurinol therapy are at risk of developing Adverse drug reactions mainly in the form of DRESS Syndrome (Drug Rash with Eosinophilia and Systemic Symptom) [6]. DRESS is an Idiosyncratic Hypersensitivity Reaction, characterized by skin rash, fever, lymph node enlargement, and multiple organ involvement. Fever, Rashes, Multiorgan involvement can be considered the Clinical Triad of DRESS Syndrome. In our patient erythematous maculopapular rash throughout the body, especially in the oral cavity and lips. It also extends to the lower limbs and upper back. The presence of excessive eosinophilia was another marker for our patient. Other studies reveal that the use of Allopurinol in Renal and Cardiovascular disease patients had a significantly higher risk of developing hypersensitivity reactions [7]. In this sense, our patient was a CKD patient and a CAD patient. Recent NSTEMI, Elevated serum urea and serum creatinine level, and Hypoalbuminemia indicate that patients had an increased chance of developing Hypersensitivity syndrome. In most of the Case Reports, 100-300 $\mathrm{mg} /$ day of Allopurinol causes DRESS Syndrome. The patient was on $100 \mathrm{mg} /$ day. In addition to the withdrawal of the offending drug Allopurinol, Steroid therapies are relevant to concern [8]. For our patient, Allopurinol was stopped on the second day of admission to the hospital while, the same day, Topical Steroid therapy began along with Supportive care. Desowen (Desonide) for local application and Kenocort $0.1 \%$ (Triamcinolone) oromucosal paste were given and continued for ten days for the follow-up. Early cessation of the offending drug and Steroid therapy results in a better outcome and alleviates the symptoms. Drug use should be monitored closely in patients with impaired renal insufficiency and liver dysfunction, to the possible risk of developing DRESS Syndrome.

\section{CONCLUSION}

Allopurinol is an FDA-approved urate-lowering agent. In general, this medication treats primarily to treat hyperuricemia, gout, and calcium nephrolithiasis. Due to the severity of potential side effects, most patients experience Fever, Eosinophilia, Multiorgan damage, or other Systemic Syndrome. The mainstay treatment for the DRESS Syndrome is the early recognition of the culprit drug and its withdrawal, coupled with steroidal therapy and supportive treatments. Understanding this rare drug reaction could potentially protect our patients and eliminate the risk of adverse drug events forever.

\section{ACKNOWLEDGEMENT}

We gratefully thank the Health Care Staff of Nirmala Medical Centre. Muvattupuzha, Kerala. India 686661

\section{FUNDING}

Nil

\section{AUTHORS CONTRIBUTIONS}

The first author (Karthika S) drafted the manuscript, the second authors (Kavya Surendran and Krishnapriya M K) collected the case and references for the manuscript, and the fourth author (Dr. Dona Maria Jetto) reviewed the manuscript.

\section{CONFLICT OF INTERESTS}

\section{Declared none}

\section{REFERENCES}

1. Markel A. Allopurinol hypersensitivity and DRESS syndrome. Am J Med 2008;121:e25-e27.

2. TA K, H A, S L, Z SA. Comparison of xanthine oxidase inhibitors in gouty patients with hyperuricemia. Asian J Pharm Clin Res 2016;9:183-6.

3. Arellano F, Sacristan JA. Allopurinol hypersensitivity syndrome: a review. Ann Pharmacother 1993;27:337-43.

4. Stamp L, Day R, Yun J. Allopurinol hypersensitivity: investigating the cause and minimizing the risk. Nat Rev Rheumatol 2015;12:235-42.

5. Chhabra M, A Gaur. Allopurinol-induced stevens-johnson syndrome and toxic epidermal necrolysis: a case report. Asian J Pharm Clin Res 2019;12:5-7.

6. Dwid NA, Cheikh MM, Mandurah AS. Allopurinol prescription patterns among patients in a Saudi tertiary care centre. J Taibah Univ Med Sci 2020;15:185-9.

7. Fathallah N, Slim R, Mokni S, Ouni B, Ghariani N, Ben Salem C. Hypersensitivity to allopurinol: a report of six cases. Clin Ther 2017;39:e2-e3.

8. Neki NS, Shergill GS, Singh A. Allopurinol induced DRESS syndrome-a case report. Int J Res Rev 2017;4:26-8. 